\title{
Clinically overweight and obese mothers and low rates of breastfeeding: Exploring women's perspectives
}

\author{
Lorna Massov ${ }^{A, B}$ MPH, RM, BA.
}

${ }^{\text {A Corresponding }}$

Author: lornachris@ paradise.net.nz

${ }^{\text {B Midwife, }}$ Wellington Hospital, $\mathrm{CCDHB}$

\begin{abstract}
:
Background: It is universally recognised that breastmilk is the best food for babies and that breastfeeding provides significant health benefits for both mothers and babies. Women who are overweight or obese have lower rates of initiation and duration of breastfeeding. There is a need to understand the woman's perspective, and any additional factors that may contribute to breastfeeding difficulties for overweight or obese women.
\end{abstract}

Objective: The aims of this research were to explore the breastfeeding experiences and perspectives of a specifically recruited group of clinically overweight and obese women and to gain an understanding of what influenced their infant feeding decisions.

Method: This study used a qualitative methodology. In-depth interviews were conducted with six mothers who were overweight or obese and initiated breastfeeding, but then did not continue to exclusively or fully breastfeed their babies. Using a thematic analysis the researcher identified seven themes which captured the breastfeeding experiences of these women.

Findings: The women believed in the importance of breastfeeding for their babies' health, but experienced challenges with latching and perceived their breast milk supply to be insufficient. The physical challenges of large breasts and body image issues when feeding in public also contributed to early cessation of exclusive breastfeeding. This study adds to the body of knowledge on this subject and to our understanding of the association between maternal overweight and obesity and early cessation of breastfeeding.

Conclusion: It is important to recognise the unique needs of overweight and obese women and provide support antenatally and postnatally. This support is crucial if we are to achieve the public health aim of reaching World Health Organization (WHO) and national targets for exclusive and full breastfeeding.

Key words: Breastfeeding, lactation, feeding behaviour, maternal obesity/body mass index (BMI), low rate

\section{INTRODUCTION}

Breast milk is considered the optimal nutrition for babies and, as a global public health strategy, the World Health Organization (WHO) recommends exclusive breastfeeding (breastmilk only with no water, other fluids or solids) for six months, with supplemental breastfeeding continuing for two years and beyond (World Health Organization, 2001). In response, the New Zealand Ministry of Health has previously set targets of exclusive and fully ${ }^{1}$ breastfeeding by $90 \%$ of mothers at six weeks postpartum by $2010,70 \%$ of mothers at three months postpartum by 2010 , and $27 \%$ of mothers at six months postpartum by 2010 (Ministry of Health, 2008).

However, key results from 2013 Plunket Society data (www. plunket.org.nz) show that the exclusive and full breastfeeding rates fall short of both international and national recommendations. In the six to nine week age group, $66 \%$ of babies are exclusively or fully breastfed and in the 10-15 week age group, 55\% of babies are exclusively or fully breastfed.
In New Zealand, between 1997 and 2012/13, the prevalence of obesity $^{2}$ among women increased from $21 \%$ to $32 \%$ (Ministry of Health, 2013). There are significant ethnic variations in the obesity pattern in New Zealand also. While $23 \%$ of Pākehā (New Zealand European) adults are obese, the Pasifika rate is triple that (68\%) and the Māori rate is double, at 48\% (Ministry of Health, 2013; Ministry of Social Development, 2010). Being overweight or obese has been identified as an independent risk factor for early cessation of breastfeeding (Li, Jewell \& GrummerStrawn, 2003; Rutishauser \& Carlin, 1992) and for reduced breastfeeding initiation rates (Krause Lovelady \& Ostbye, 2011; Li et al., 2003). This association between mothers being overweight and low rates of breastfeeding initiation and duration is therefore of public health concern given that the prevalence of obesity among child-bearing women is increasing worldwide (Vahratian, 2009).

However, the reasons mothers who are overweight are less likely to initiate breastfeeding, and more likely to breastfeed for a shorter

${ }^{1}$ The infant has taken breastmilk only. No other liquids or solids have been given except a minimal amount of water or prescribed medicines in the last 48 hrs (this matches the WHO exclusive indicator).

2. The World Health Organization defines a Body Mass Index (BMI) >25 as clinically overweight, and a BMI >30 obese (World Health Organization, 2000) 
duration, are not clear. Breastfeeding behaviour is complex and multifactorial (Amir \& Donath, 2007), and a woman's decision and ability to breastfeed are influenced as much by emotional and psychological responses as by physiological responses (Amir \& Donath, 2007; Rasmussen \& Maher, 2007.

\section{Studies of populations in the \\ United States and elsewhere have found that overweight and obese mothers were less likely to initiate breastfeeding compared to normal weight women.}

Research on the negative association between maternal overweight and obesity and breastfeeding is predominantly quantitative. Studies of populations in the United States (Kitsantas \& Pawloski, 2009; Li et al., 2003) and elsewhere (Baker, Michaelsen, Sorensen \& Rasmussen, 2007; Donath \& Amir, 2008; Guelinckx, Devlieger, Bagaerts, Pauwels \& Vansant, 2011; Mok et al., 2008) have found that overweight and obese mothers were less likely to initiate breastfeeding compared to normal weight women, and that they ceased breastfeeding earlier than normal weight women.

While these studies have found an association between maternal overweight and initiation and duration of breastfeeding, they are unable to address the question of why. It is not clear which factors influence a mother who is overweight or obese to not initiate breastfeeding or to breastfeed for a shorter time. The causes are likely to be multifactorial (Lovelady, 2005), and may be related to physiological factors (e.g., hormonal variations), physical factors (e.g., large breasts) and socio-cultural factors (e.g., body image, low self-esteem, poor mental health) (Amir \& Donath, 2007).

\section{Delayed onset of lactation and poorer prolactin response to suckling are associated with maternal overweight/obesity.}

Delayed onset of lactation and poorer prolactin response to suckling are associated with maternal overweight/obesity (Dewey, Nommsen-Rivers, Heinig \& Cohen; Nommsen-Rivers et al., 2010; Chapman \& Perez-Escamilla, 1999; Hilson, Rasmussen $\&$ Kjolhede, 2004). In a population study of 40 mothers with full-term infants, Rasmussen \& Kjolhede (2004) measured serum prolactin and progesterone concentration in normal weight and overweight women in the first week postpartum. Women who were overweight or obese had a lower prolactin response to suckling, thus compromising their ability to produce milk, which over time could result in early cessation of breastfeeding. While this finding provides evidence of a biological basis for the association between maternal overweight/obese and shorter duration of breastfeeding it is important to note that the prolactin response is dependent on effective suckling by the baby. If overweight or obese women have greater latching difficulties, the prolactin stimulus is further reduced, thus additionally affecting milk production and supply.

There are a number of risk factors for delayed onset of lactation. These include caesarean birth, prolonged second stage of labour, gestational diabetes mellitus (Chapman \& Perez-Escamilla, 1999; Chen, Nommsen-Rivers, Dewey \& Bo, 1998; Dewey et al., 2003; Perez-Escamilla \& Chapman, 2001) and large for gestational age (LGA) babies (Rasmussen \& Maher, 2007). Maternal obesity carries with it a greater risk for emergency caesarean section, instrumental birth, shoulder dystocia and gestational diabetes mellitus (Perez-Escamilla \& Chapman, 2001; Cedergreen, 2004).

Physical and mechanical factors present difficulties to successful latching of the baby onto the breast. Obese women tend to have larger breasts, with an excess of periareolar adipose tissue which may flatten the areola and nipple, making the nipple more difficult for the newborn to grasp. This difficulty contributes to the reduced prolactin response to suckling (Coates, 1989; Jevitt, Hernandez \& Groer, 2007; Lovelady, 2005).

Additionally, women's experience of breastfeeding is influenced by a range of psychological, emotional and sociocultural processes. These cognitive factors have been found to influence breastfeeding behaviour and breastfeeding duration. Psychological factors statistically associated with the duration of breastfeeding include: anxiety, breastfeeding expectations, faith in breastmilk, and breastfeeding self-efficacy (O'Brien, Buikstra \& Hegney, 2008).

Higher maternal anxiety is associated with early weaning (Papinczak \& Turner, 2000) and maternal postnatal depression has a significant negative impact on breastfeeding duration (Dunn, Davies, McCleary, Edwards \& Gaboury, 2006; Henderson, Evans, Straton, Priest \& Hagan, 2003; Taveras et al., 2003). Further, there is a well-documented association between obesity, depression and anxiety in women. A recent study (LaCoursiere, Baksh, Bloebaum \& Varner, 2006) found a two-fold increase in self-reported postpartum depressive symptoms requiring assistance among overweight and obese women compared to normal weight women.

\section{Low maternal self-esteem and self- confidence, and reduced belief in their ability to breastfeed are two of the strongest predictors of breastfeeding cessation.}

Low maternal self-esteem and self-confidence, and reduced belief in their ability to breastfeed are two of the strongest predictors of breastfeeding cessation (Blyth et al., 2002; Grassley \& Nelms, 2008; Harris, Nayda \& Summers, 2003). Low maternal selfesteem is associated with negative body image and pregnant women may be concerned about changes in their body shape. Obesity is also linked to poor body image, particularly for women (Rubin, 1984). Hilson et al. (2004) found that obese women were less satisfied with their appearance and planned to breastfeed for a shorter duration than normal weight women. This dissatisfaction with body image may extend into embarrassment with exposure of their body (Amir \& Donath, 2007) and is likely to be one reason overweight and obese mothers feel uncomfortable breastfeeding in public.

A frequent major breastfeeding problem identified by women was perceived low milk supply (Lamontagne, Hamelin \& StPierre, 2008). Fewer obese mothers felt that their milk supply was sufficient at one month and three months compared to normal weight women (Mok et al., 2008). This challenge has been recognised with some midwifery care plans identifying overweight and obese women as needing extra support due to their reduced perceptions of breast fullness and milk production (Jevitt et al., 2007).

The importance of health professional support for women in establishing and sustaining breastfeeding is widely recognised (Glover, Waldon, Manaena-Biddle, Holdaway \& Cunningham, 2009; Guyer, Millward \& Berger, 2012; Thulier \& Mercer, 2009). 
Research examining lactation-counselling practices used with obese mothers (Rasmussen, Lee, Ledkovsky \& Kjolhede, 2006) found that the majority of health care providers surveyed neither believed that there was a difference in the success rate between obese mothers and normal weight mothers, nor advised obese mothers differently about breastfeeding (Rasmussen et al., 2006).

\section{The importance of health professional support for women in establishing and sustaining breastfeeding is widely recognised.}

Qualitative research can explore women's breastfeeding experiences in more depth and examine the influence of psychological and/ or behavioural factors, (Rasmussen \& Maher, 2007). However, a literature search was unable to find any qualitative research which specifically studied overweight and obese mothers and their experiences of breastfeeding. The important issue is whether these personal factors affect overweight and obese women differently to normal weight women (Rasmussen \& Maher, 2007).

The aims of this research were to describe, from clinically overweight and obese women's perspectives, their experiences of breastfeeding; what influenced their infant feeding decisions; and to understand better the reasons for short breastfeeding duration among this group.

The following research question was formulated: 'what factors for short breastfeeding duration can be identified from the experiences of a small group of clinically overweight and obese women?'

\section{METHOD}

A qualitative design using thematic analysis was chosen to explore the breastfeeding experiences of six clinically overweight mothers who did not exclusively breastfeed their infants. Thematic analysis is a method for identifying, analysing and reporting patterns or themes within data. It describes the data set in "rich" detail (Braun $\&$ Clarke, 2006) and has the potential to interpret various aspects of the research topic (Boyatzis, 1998), and to report experiences, meanings and the reality of participants (Braun \& Clarke, 2006).

Rigorous qualitative research acknowledges that researchers are part of the setting, context and culture they are trying to study (Rice \& Ezzy, 1999). My professional role as a practising midwife was made clear to the participants and supported my understanding of the nature of the breastfeeding difficulties the participants experienced. This knowledge guided me when interviewing and assisted in developing a rapport with the women.

\section{Participants}

Six women from a large city in New Zealand were recruited for the study. The inclusion criteria were: 1) women who were clinically overweight or obese at the time of their antenatal booking visit (as defined using the WHO definitions of - BMI $>25$ clinically overweight, BMI $>30$ obese (World Health Organization, 2000)) and: 2) women who had initiated breastfeeding but were not exclusively or fully breastfeeding at four to six weeks postpartum. The women's ages ranged from 31-36 years. Four of the women identified as New Zealand European or Pākehā, one as Samoan and one as New Zealand Māori. Two of the participants were primiparous and four were multiparous. Two of the women interviewed had had normal vaginal births, while four of the women had had caesarean section births. Two of the six participants had had gestational diabetes. The participants' BMIs, collected by their Lead Maternity Carers at the antenatal booking visit, were 35, 36, 40, 43, 46 and 66 .

Ethical approval was obtained from the University of Otago Ethics Committee (Reference Code: 13/029). The women who met the study criteria were recruited by Lead Maternity Carer (LMC) midwives in the community and core midwives in local hospitals. The midwives identified eligible participants, outlined the project and gave them an information sheet and a consent form. Interested participants were phoned by the researcher who explained the research project further and arranged an interview time. Signed consent for a digitally recorded interview was obtained before the interviews commenced. An information sheet was provided and explained that participants could withdraw at any time, without prejudice. Pseudonyms were allocated to the women participants to preserve confidentiality.

The women were interviewed in a semi-structured interview, lasting 30 minutes to an hour, in their own home. In-depth interviewing is appropriate to qualitative research as it allows participants to describe what is meaningful and important to them using their own words (Minichiello, Aroni \& Hays, 2008). The interview opened with a broad question: 'Could you tell me about your breastfeeding experience from the moment your baby was born until the present?' This key question enabled the mother to tell her breastfeeding story, including reflecting on any difficulties encountered, and to highlight what was significant for her.

\section{Data Analysis}

The interview recordings were transcribed verbatim by the researcher with initial themes in the narratives being identified during the transcription process. Seven main themes emerged: breastfeeding as difficult, perceived insufficient breastmilk supply, physical difficulties-latching and mechanical factors, unrealistic expectations, pressure to breastfeed, professional help as distressing, and being philosophical about their decision.

\section{FINDINGS}

Four of the six women intended to exclusively breastfeed their babies immediately after birth for as long as possible. One of the women had planned to bottle-feed her baby after birth but was persuaded by her LMC midwife to attempt exclusive breastfeeding. One of these women had planned to mix feed, i.e., breast and bottle feeding combined. The overarching theme that emerged in the participants' narratives was that breastfeeding was a challenging and demanding experience that required practice and perseverance. As well as the physical challenges of breastfeeding, the experiences of the women evoked complex emotional responses highlighting the integral relationship between breastfeeding and emotional wellbeing. The seven main themes are illustrated using direct quotes.

\section{Breastfeeding as Difficult}

Rather than finding it a natural instinctive process, the women in this study described their experiences of breastfeeding in terms of being difficult, a struggle and a challenging task that had to be worked at to get right. Breastfeeding was conceptualised as "work" or "labour", or a task needing preparation in order to be successful.

Yes well I think for us it was breastfeeding is good for him, it's free and it's a nice bonding thing but I can't say it's a bonding thing now. For me it feels really hard, it feels like a full time job... (Kate)

...so I had to use a nipple shield anyway which is kinda difficult, you know, it's not just a matter of getting it out and away you go. You know there's still a lot of prep work. 
I guess you'd say, because you still have to make sure it's sanitised and on properly and so on and so forth. It was still a lot of work. (Jess)

The women expressed a concern that the closeness created by breastfeeding was stifling and confining. There was a sense of loss of autonomy and self-identity for the women due to the demands of breastfeeding. For Annie there was a sense that introducing bottles enabled some separation from her infant and regaining of control over her life. This allowed her partner to assist in feeding and for them to establish predictable feeding routines:

... when you are solely breastfeeding, it's like he got really attached to me and he didn't want to let me go.... but now that he has bottles it's like, well can you feed him while I do this whereas if I was breastfeeding it's a little bit harder to let him get attached or when I was leaving him it would have been very, very hard.... (Annie)

\section{Insufficient Breastmilk Supply}

All the women attributed lack of breastfeeding success and introduction of formula as being at least partly due to insufficient breastmilk supply. This unavoidable conclusion was strongly linked to their baby's discontented behaviour and to assessments of their baby's weight loss by health professionals:

...then because I didn't have enough milk they realised he wasn't getting enough because he was screaming half the time and he wasn't putting on the weight... He was getting so frustrated and so upset, so that would get me upset which doesn't really help with the "let down"... (Annie)

We were in bed because he just cried all night and he wouldn't sleep and he wouldn't settle. I thought he was cluster feeding. I thought he was making up for the time he didn't get much at the breast and then it turned out that he was actually starving...(Kate)

For many of the women, once they had decided to introduce supplements for their baby, the change in their baby's behaviour and weight increase seemed to confirm their belief that they had had insufficient breast milk supply and they felt vindicated:

.....so he's been fully bottle fed for at least a week and he's been so much happier and when Plunket came and did their first visit he'd put on the most weight the entire time so it makes me feel like he was just never getting enough. (Jess)

One woman talked about her milk coming in "late". As she stated "...they had to put him straight on to formula because my milk didn't come in until two days after I'd given birth". Additionally some women were concerned because they couldn't feel any breast changes which are often a sign of "milk coming in" or the "let down."

"...my boobs never felt full or hard and by that stage I'd moved on to expressing and I just noticed that I wasn't getting, I mean $20 \mathrm{mls}$ each feed would have been my best take. (Rowan)

\section{Physical Difficulties - Latching and Mechanical Factors}

Five of the six women interviewed had difficulties latching their babies related to the physical challenges of having large breasts. For example, Rowan attributed her difficulty with breastfeeding to her increased body weight and the size of her breasts. That is, she directly attributed her lack of success in breastfeeding to her weight, or at least felt it was a significant factor:

Yes, definitely, I feel it was, the lactation consultant said "lovely big boobs, you shouldn't have any problems" but no, it didn't help with milk production, or the technical side of things.

The women described their breasts in terms of their heaviness, and of the weight of them pressing on their baby; in some instances objectifying them as if separating them from their bodies:

They were very full and I'm a big busted girl, they just felt like this huge weight... and because I'm a big busted girl having him in the right position... In the end I found the rugby position the best because I didn't have to worry about the fact of squishing him to death... (Annie)

Probably, because my boobs had just gone boing (gestures with her hands over her breasts like they had gotten bigger), like really big, I didn't know whether he was breathing. (Carol)

The size of the women's breasts also posed practical problems with latching their babies and made it difficult to feed discreetly in public. Comments associated with this aspect included "selfconsciousness", "discomfort", and "reluctance" to breastfeed in front of others:

Yes, me personally, I'm just too self-conscious to, because they're so big, to actually get them out in public. (Jess)

... Yeah I guess because there's so much boob there and also I didn't want it to become a problem. I did want to feed and everything but I did notice there was an uncomfortableness about it for me. (Rowan)

\section{Unrealistic Expectations}

All the women found their breastfeeding experience difficult and challenging. There was a real sense that they weren't prepared for the reality of breastfeeding. They suggested that there was almost a conspiracy of secrecy around the realities of breastfeeding and the difficulties many women encounter.

...like, I think it's hard because everyone says that breastfeeding is natural so when you can't do it you feel unnatural and no-one really talks about how hard it is... no, everyone talks about how easy it is, how natural it is or that it takes practice, it comes with ease, but no-one talks about the actual sheer exhaustion of it, you know... (Kate)

I know that they do put a lot of emphasis on breastfeeding but it's hard and I think they should put more emphasis on that and just how long it can take to establish. (Rowan)

One woman reflected on the unrealistic expectations of the women in her antenatal class about breastfeeding and newborn baby behaviour:
Most of the women had never been around children before and their whole expectations were "I'm going to be able to breastfeed my kids, they're not going to cry, they're going to be settled within seconds, I'm just going to look at them and they're going to be, like..." And I was, like, "whoa, rewind that, realistic slap in the face here". (Annie)

Annie believed these (so-called "unrealistic") women were influenced by idealised media images:

Oh, you know, they think of babies they see on TV; they're well-behaved; they're cute; they hardly ever cry. Hello, real world! (Annie).

One woman wanted to hear from other women about the realities of breastfeeding, including the difficulties and problems they experienced. 
...the ones that do have children are really, like: "Yeah, it is really tough", and I'm, like: "why didn't you tell me that before?" (Kate)

\section{Pressure to Breastfeed}

Two of the women described encountering a judgmental attitude from people about bottle-feeding following their decision to either supplement or wean their babies. The women also spoke of the pressure to breastfeed that is put on women by society and, also, by themselves:

\begin{abstract}
There is a lot of pressure just in general society to breastfeed, a lot of it. Even if you can't breastfeed for medical reasons, you know, there is a lot of pressure out there to breastfeed. I think mothers put a lot of pressure on themselves and cause a lot of problems to themselves because they can't do it or they feel bad because they supplement their babies... I think we put a lot of pressure on ourselves to do it. (Jess)
\end{abstract}

...I think its peer pressure to perform as a good mother...I did feel pressured to be a good mother and that and, like, you should breastfeed. (Penny)

\section{Professional Help as Distressing}

For one woman the inappropriate behaviour of one midwife was distressing. She found the experience of having this midwife assist with breastfeeding aggressive and rough:

I just remember the midwife coming in and almost angry
that I was upset because I was having trouble doing it and
"I'll show you how to express" but then just pretty much
grabbed my breast without asking if that was OK. And
being really, really rough and aggressive with her and I
kind of...it's not on... She just ruined the whole stay in
hospital, like then it made me apprehensive about any other
midwife that walked in the room and what were they going
to do to me? And stuff... (Jess)

For another woman the experience was just as disempowering. The woman commented that rather than assisting her by showing her how to attach her baby to the breast, the midwives were taking over.

\section{I felt really disempowered totally because I felt like every time I pressed the button I was a problem that needed sorting, not a person who needed help. (Kate)}

\section{Being Philosophical about their Decision}

Despite the breastfeeding difficulties they experienced, the women acknowledged the benefits of breastfeeding and were pleased that they had persevered and given their baby this optimum nutrition in the beginning:
...it's up to you to switch to formula but try breastfeeding because all the nutrients come from the milk. I always think it's the best for babies. (Carol)

\section{I would do it again in a heartbeat. I would not not do it. I would spend hours pumping if that's what I've got to do. I'd do it because it's best for a child; because it's the good stuff right away. (Annie)}

The women rationalised their decisions to introduce supplementary feeding in terms of the importance of their baby's health and wellbeing:

...at the end of the day if baby is happy and healthy that
should be all that matters. We all know that breast is best
but for some women it doesn't work. (Jess)

\section{DISCUSSION}

The aims of this research were to hear from this small group of women who were overweight or obese, to learn about their experiences of breastfeeding and to report what influenced their decisions about initiation and cessation of breastfeeding. The specific explorations of difficulties with breastfeeding largely aligned with other related research. These were the challenging nature of breastfeeding, which included perceived insufficient breastmilk supply, and latching difficulties. For women in this study there was more of an emphasis on the physical difficulties of latching a baby on to large breasts than that found elsewhere in the literature. Large breasts were also an issue in terms of discretion when breastfeeding in public and a body image concern for these women. Other findings included cognitive factors, such as unrealistic expectations, and societal pressure to breastfeed. However, the women were philosophical about their infant feeding decisions. Concerning was the distress caused for some of the women by the approach and actions of some midwives.

\section{The women in this study described their breastfeeding experiences in terms of work, hardship and struggle.}

The women in this study described their breastfeeding experiences in terms of work, hardship and struggle. These findings are similar to those of Larsen, Hall and Aagaard (2008), Schmied and Barclay (1999) and Hjalmhult and Lomborg (2012), whose studies included women who described their breastfeeding experiences as a "battleground" or "a fight", or breastfeeding itself as sustained hardship and their having to fight to succeed.

These struggles possibly linked to having perceived insufficient breastmilk supply which contributed to early cessation of breastfeeding. For five out of the six women this problem with supply was thought to be due to difficulties latching their babies on to their breast. The infant's ability to latch and suck effectively has a physiological influence on breastmilk supply (Woolridge, 1995). These physical factors were found to be associated with early cessation of feeding in studies by McFadden and Toole (2006) and Binns andle Scott (2002).

Binns and Scott (2002) also found that women diagnosed breastmilk insufficiency on the basis of their baby's behaviour. This included references to a baby's unsettled behaviour, frequent crying, screaming and reluctance to sleep. Whereas after the introduction of supplements the women described the change in their baby's behaviour as - more settled "and happier". Of concern for some of the women in this current study was their baby's weight gain. This concern with weight is also reflected in other research, for example Hjalmhult and Lomborg (2012) in their study, who describe women beaming with pride when the baby's weight increased.

The issues described above may well be experienced by women who are not overweight or obese. However, in this study, the women had additional physical challenges related to their body and breast size plus issues of body image, particularly when attempting to feed in public. Five of the women in this study, who experienced difficulties with latching their baby to the breast, attributed their difficulties to the size of their breasts. This supports the idea that mechanical difficulties are one reason overweight and obese women have low rates of initiation and duration of breastfeeding. One woman directly attributed her breastfeeding difficulties to both her body size and to the size of her breasts. 
Although some of the women in this study reported positive experiences with health professionals, three women found the way health professionals touched their bodies distressing, uncomfortable and disempowering. The women used strong terms such as "manipulate", "shoving" and "aggressive" to describe the actions of midwives as they attempted to latch the baby to the mother's breast. This differed from the literature reviewed, which described professional help to breastfeed. For example, participants in Lamontagne, Hamelin and St-Pierre's study (2008) found that health care professionals could sometimes be a negative influence when their breastfeeding advice to women was inconsistent, inadequate, or in conflict with the advice of others.

\section{LIMITATIONS OF THE STUDY}

This study has explored the experiences and perspectives of six women and the difficulties they encountered when initiating breastfeeding. It seeks to deepen our understanding of the issues facing overweight and obese women when they initiate breastfeeding. A limitation of this study is the small sample size. Thus the results are not generalisable to the wider population of women who are overweight or obese.

\section{IMPLICATIONS FOR PRACTICE}

Managing weight gain in pregnancy may contribute to breastfeeding success. However, there is a limited time available for midwives to assist women to make lifestyle changes during pregnancy and postpartum. For maximum effectiveness these changes need to begin pre-conceptually and at least by the time of the first antenatal visit ( $\mathrm{Li}$ et al., 2003; Hilson, Rasmussen \& Kjolhede, 2006). Antenatal education should also include healthy eating guidelines with women being encouraged to avoid gaining more than the recommended weight gain during pregnancy. Importantly, women should never be advised to lose weight during pregnancy (Hilson et al., 2006).

\section{Women who are overweight or obese need additional breastfeeding support in the antenatal period, immediately after birth and postnatally.}

Women who are overweight or obese need additional breastfeeding support in the antenatal period, immediately after birth and postnatally. There are unique challenges for this group of women, such as reduced prolactin stimulation, body shape and image issues and physical difficulties due to large breasts. Being aware of these issues and planning supportive care have the potential to enable this group of women to achieve a positive and successful breastfeeding experience, increased self-esteem and confidence, and better health for their babies.

\section{CONCLUSION}

This study gives voice to six overweight and obese women, their experiences of breastfeeding and what influenced their infant feeding decisions. The women report that their breastfeeding experiences were commonly problematic and challenging despite a strong desire to breastfeed and an awareness of its importance to their baby's health. Women in this study experienced breast latching difficulties which could have been a contributing factor in a perceived insufficient breastmilk supply. These factors may also be common for women who are not overweight or obese but in this study the women identified the physical factor of large breasts as contributing to their breastfeeding difficulties in addition to body image issues, particularly when attempting to breastfeed in public. The findings highlight the need for overweight and obese women to have additional and specific breastfeeding support during childbirth.

\section{...the complex nature of breastfeeding means that additional factors contribute to the difficulties that overweight and obese women face when establishing breastfeeding.}

While quantitative studies show a strong biological association between maternal overweight and obesity and low rates of initiation and duration of breastfeeding, the complex nature of breastfeeding means that additional factors contribute to the difficulties that overweight and obese women face when establishing breastfeeding. It is these potentially modifiable factors which midwives, other health professionals and women's social support networks can positively influence to maximise the possibility that this group of women will succeed at breastfeeding.

\section{ACKNOWLEDGEMENTS}

The author would like to thank all the women who participated in this research project and who so generously shared their time and experiences. Thanks also to my midwifery colleagues, both within the hospital and the community, who assisted me with recruitment of participants and for their encouragement and advice. This research project was undertaken as a partial requirement towards a Masters of Public Health at the University of Otago, Wellington. No funding was received for this project. There was no conflict of interest.

\section{REFERENCES}

Amir, L. H., \& Donath, S. (2007). A systematic review of maternal obesity and breastfeeding intention, initiation and duration. BMC Pregnancy and Childbirth, 10(9),1-9.

Baker, J., Michaelsen, K., Sorensen, T., \& Rasmussen, K. (2007). High prepregnant body mass index is associated with early termination of full and any breastfeeding in Danish women. American Journal Clinical Nursing, 86, 404-11.

Binns, C. W., \& Scott, J.A. (2002). Breastfeeding: Reasons for starting, reasons for stopping and problems along the way. Breastfeeding Review, $10(2), 1319$.

Blyth, R., Creedy, D., Dennis, C-L., Moyle, W., Pratt, J., \& De Vries, S. M. (2002). Effect of Maternal Confidence on Breastfeeding Duration: An Application of Breastfeeding Self-Efficacy Theory. Birth, 29(4),278-84.

Boyatzis, R.E. (1998). Transforming Qualitative Information. Thematic Analysis and Code Development. California, London, New Delhi: Sage Publications.

Braun, V., \& Clarke, V. (2006). Using thematic analysis in psychology. Qualitative Research in Psychology, 3, 77-101.

Cedergreen, M. (2004). Maternal Morbid Obesity and the Risk of Adverse Pregnancy Outcome. The American College of Obstetricians and Gynecologists, 103(2), 219-24.

Chapman, D. J., \& Perez-Escamilla, R. (1999). Identification of risk factors for delayed onset of lactation. Journal of the American Dietetic Association, 99(4), 450-454.

Chen, D., Nommsen-Rivers, L., Dewey, K., \& Bo, L. (1998). Stress during labor and delivery and early lactation performance. American Journal Clinical Nutrition, 68, 335-44.

Coates, M. (1989). Assisting the Newborn to Latch On to the Very Large Breast. Journal Human Lactation, 5(3),131-2. 
Dewey, K., Nommsen-Rivers, L., Heinig, J. \& Cohen, R. (2003). Risk Factors for Suboptimal Infant Breastfeeding Behaviour, Delayed Onset of Lactation and Excess Neonatal Weight Loss. Paediatrics, 112, 607-19.

Donath, S., \& Amir, L. H. (2008). Maternal obesity and initiation and duration of breastfeeding: data from the longitudinal study of Australian children. Maternal and Child Nutrition, 4, 163-70.

Dunn, S., Davies, B., McCleary, L., Edwards, N., \& Gaboury, I. (2006). The Relationship Between Vulnerability Factors and Breastfeeding Outcome. JOGNN, 35(1), 87-97.

Glover, M., Waldon, J., Manaena-Biddle, H., Holdaway, M., \& Cunningham, C. (2009). Barriers to Best Outcomes in Breastfeeding for Māori: Mothers' Perceptions, Whānau Perceptions, and Services. Journal Human Lactation, 25(3), 307-16.

Grassley, J. S., \& Nelms, T. P. (2008). Understanding Maternal Breastfeeding Confidence: A Gadamerian Hermeneutic Analysis of Women's Stories. Health Care for Women International, 29, 841-62.

Guelinckx, I., Devlieger, R., Bogaerts, A., Pauwels, S., \& Vansant, G. (2011). The effect of pre-pregnancy BMI on intention, initiation and duration of breastfeeding. Public Health Nutrition, 15(5), 840-8.

Guyer, J., Millward, L.J., \& Berger, I. (2012). Mothers' breastfeeding experiences and implications for professionals. British Journal of Midwifery, 20(10), 724-33.

Harris, M., Nayda, R., \& Summers, A. (2003). Breasts and breastfeeding: Perspectives of women in the early months after birthing. Breastfeeding Review, 11(3), 22-9.

Henderson, J. J., Evans, S.F., Straton, J., Priest, S. R., \& Hagan, R. (2003). Impact of Postnatal Depression on Breastfeeding Duration. Birth, 30, 175-80.

Hilson, J. A., Rasmussen, K. M., \& Kjolhede, C. L. (2004). High Prepregnant Body Mass Index is Associated With Poor Lactation Outcomes Among White, Rural Women Independent of Psychosocial and Demographic Correlates. Journal Human Lactation, 20(1), 18-29.

Hjalmhult, E., \& Lomborg, K. (2012). Managing the first period at home with a newborn: a grounded theory study of mothers' experiences. Scandinavian Journal of Caring Sciences, 26, 654-62.

Jevitt, C., Hernandez, I., \& Groer, M. (2007). Lactation Complicated by Overweight and Obesity: Supporting the Mother and Newborn. Journal of Midwifery \& Women's Health, 52(6), 606-13.

Kitsantas, P., \& Pawloski, L.R. (2009). Maternal obesity, health status during pregnancy, and breastfeeding initiation and duration. The Journal of Maternal-Fetal and Nutritional Medicine, 23(2), 135-41.

Krause, K., Lovelady, C., \& Ostbye, T. (2011). Predictors of Breastfeeding in Overweight and Obese Women: Data from Active Mothers Postpartum (AMP). Maternal Child Health Journal, 15, 367-75.

LaCoursiere, D. Y., Baksh, L., Bloebaum, L., \& Varner, M. W. (2006). Maternal Body Mass Index and Self-Reported Postpartum Depressive Symptoms. Maternal Child Health Journal, 10(4), 385-90.

Lamontagne, C., Hamelin, A-M., \& St-Pierre, M. (2008). The breastfeeding experience of women with major difficulties who use the services of a breastfeeding clinic: a descriptive study. International Breastfeeding Journal, 3(17), 1-13.

Larsen, J. S, Hall, E. O. C., \& Aagaard, H. (2008). Shattered expectations: when mothers' confidence in breastfeeding is undermined a metasynthesis. Scandinavian Journal of Caring Sciences, 22(4), 653-61.

Li, R., Jewell, S., \& Grummer-Strawn, L. (2003). Maternal obesity and breastfeeding practices. American Journal Clinical Nursing, 77, 931-6.

Lovelady, C. A. (2005). Is Maternal Obesity a Cause of Poor Lactation Performance? Nutrition Reviews, 63(10), 352-5.

McFadden, A., \& Toole, G. (2006). Exploring women's views of breastfeeding: a focus group study within an area with high levels of socio-economic deprivation. Maternal and Child Nutrition, 2, 156-68.

Minichiello, V., Aroni, R., \& Hays, T. (2008). In-depth interviewing: Principles, Techniques, Analysis. 3rd edition. Australia: Pearson Education.

Ministry of Health. (2008). National Advisory Committee on Breastfeeding. Protecting, Promoting and Supporting Breastfeeding in New Zealand.

Ministry of Health. (2012/13). New Zealand Health Survey. Annual update of key findings 2012/13. Retrieved from http://www. health.govt.nz
Ministry of Social Development. (2010). The Social Report. 2010. Retrieved from http://www.socialreport.msd.govt.nz

Mok, E., Multon, C., Piguel, L., Barroso, E., Goua, V., \& Christine, P. (2008). Decreased Full Breastfeeding, Altered Practices, Perceptions, and Infant Weight Change of Prepregnant Obese Women: A Need for Extra Support. Pediatrics, 121(5), 1319-24.

Nommsen-Rivers, L., Chantry, C., Peerson, J., Cohen, R., \& Dewey, K. (2010). Delayed onset of lactogenesis among first-time mothers is related to maternal obesity and factors associated with ineffective breastfeeding. American Society for Nutrition, 92, 574-84.

O'Brien, M., Buikstra, E., \& Hegney, D. (2008). The influence of psychological factors on breastfeeding duration. Journal of Advanced Nursing, 63(4), 397-408.

Papinczak, T. A., \& Turner, C. T. (2000). An analysis of personal and social factors influencing initiation and duration of breastfeeding in a large Queensland maternity hospital. Breastfeeding Review, 8(1), 25-33.

Perez-Escamilla, R., \& Chapman, D. (2001) Validity and Public Health Implications of Maternal Perception of the Onset of Lactation: An International Analytical Overview. The Journal of Nutrition. 131(11), 3021-4

Rasmussen, K. M., Lee, V. E., Ledkovsky, T.B., \& Kjolhede, C. L. (2006). A Description of Lactation Counseling Practices That Are Used With Obese Mothers. Journal Human Lactation, 22(3), 322-7.

Rasmussen, K.M., \& Maher, K. (2007). Association of Maternal Obesity Before Conception with Poor Lactation Performance. Annual Review Nutrition, 27, 103-21.

Rice, P L., \& Ezzy, D. 1999). Qualitative Research Methods. A Health Focus. Oxford: Oxford University Press.

Royal New Zealand Plunket Society. (2010). Plunket Breastfeeding Data - Analysis of 2004-2009 Data. Royal New Zealand Plunket Society data 2013. www.plunket.org.nz

Rubin, R. (1984). Maternal Identity and the Maternal Experience. New York: Springer.

Rutishauser, I., \& Carlin, J. B. (1992). Body mass index and duration of breastfeeding: a survival analysis during the first six months of life. Journal of Epidemiology and Community Health, 46, 559-65.

Schmied, V., \& Barclay, L. (1999). Connection and Pleasure, Disruption and Distress: Women's Experience of Breastfeeding. Journal Human Lactation, 15(4), 325-33.

Taveras, E. M., Capra, A. M., Braveman, P. A., Jensvold, N.G., Escobar, G. J., \& Lieu, T. A. (2003). Clinican Support and Psychosocial Risk Factors Associated With Breastfeeding Discontinuation. Paediatrics, 112(1), 108-14.

Thulier, D., \& Mercer, J. (2009). Variables Associated with Breastfeeding Duration. JOGNN, 38, 259-68.

Vahratian, A. (2009). Prevalence of Overweight and Obesity Among Women of Child-Bearing Age: Results from the 2002 National Survey of Family Growth. Maternal Child Health Journal, 13, 268-273.

Woolridge, M. (1995). Breastfeeding: Physiology into practice. In: Davies D, Editor. Nutrition in Child Health, 13-31. London: Royal College of Physicians Press.

World Health Organization. (2000). Obesity: Preventing \& Managing the Global Epidemic. Geneva.

World Health Organization. (2001). Expert consultation on the optimal duration of exclusive breastfeeding. Geneva.

\section{Accepted for publication May 2015}

Massov, L. (2015). Clinically overweight and obese mothers and low rates of breastfeeding: Exploring women's perspectives. NZCOM Journal 51, 23-29.

http://dx.doi.org/10.12784/nzcominl51.2015.4.23-29 